\title{
Low Degree Euclidean and Minkowski Pythagorean Hodograph Curves
}

\author{
Zbyněk Šír ${ }^{1}$ and Jiří Kosinka ${ }^{2}$ \\ 1 Faculty of Mathematics and Physics, Charles University in Prague, \\ Sokolovská 83, 18675 Prague, \\ email: zbynek.sir@mff.cuni.cz \\ 2 Centre of Mathematics for Applications, Department of Informatics, University of Oslo, \\ PO Box 1053, Blindern, 0316 Oslo, Norway, \\ email: jiri.kosinka@email.cz
}

\begin{abstract}
In our contribution we study cubic and quintic Pythagorean Hodograph $(\mathrm{PH})$ curves in the Euclidean and Minkowski planes. We analyze their control polygons and give necessary and sufficient conditions for cubic and quintic curves to be PH. In the case of Euclidean cubics the conditions are known and we provide a new proof. For the case of Minkowski cubics we formulate and prove a new simple geometrical condition. We also give conditions for the control polygons of quintics in both types of planes.

Moreover, we introduce the new notion of the preimage of a transformation, which is closely connected to the so-called preimage of a PH curve. We determine which transformations of the preimage curves produce similarities of $\mathrm{PH}$ curves in both Euclidean and Minkowski plane. Using these preimages of transformations we provide simple proofs of the known facts that up to similarities there exists only one Euclidean PH cubic (the so-called Tschirnhausen cubic) and two Minkowski PH cubics. Eventually, with the help of this novel approach we classify and describe the systems of Euclidean and Minkowski PH quintics.
\end{abstract}

\section{Introduction}

Pythagorean Hodograph (PH) curves (see the survey [15], the book [17] and the references cited therein) form an interesting subclass of polynomial parametric curves. They possess a piecewise polynomial arc length function and rational offset curves in the planar case. PH curves provide an elegant solution to various difficult problems occurring in many applications, in particular in the context of CNC (computer-numerical-control) machining and offset approximations by rational splines. The notion of PH curves was also generalized to Minkowski plane and space with applications to offsets trimming and medial axis transform approximations.

Since their introduction in [10] planar PH curves have been studied in many publications. The greatest attention was devoted to various construction techniques $[2,9,12$, $13,20,22,28,30]$. The number of publications devoted to structural and classification results is much smaller. The original publication [10] and later [1] studying PH curves from the point of view of complex numbers seem to be the most important. In [34] the conditions for the monotonicity of curvature were studied for $\mathrm{PH}$ quintics. 
Spatial PH curves were introduced in [11], and they have later been characterized using results about Pythagorean quadruples in the ring of polynomials and quaternion calculus $[7,14]$. Various construction and other techniques are also available $[4,16,18$, $21,32,33]$.

In the Minkowski setting, Minkowski Pythagorean hodograph (MPH) curves were introduced by [29]. They are important in the context of the medial axis transform and approximation of planar domain boundaries and their offsets. MPH curves and their applications were studied in [5, 24, 25, 32]. In [23] a classification of planar and spatial MPH cubics was given. The importance of planar Minkowski curves consist in the fact that they (when embedded in the Minkowski space) describe certain symmetric domains.

In [6] a unified approach to PH and MPH curves in planar and spatial settings based on Clifford algebra calculus was proposed.

In this paper we analyze the types and properties of control polygons of $\mathrm{PH}$ cubics and quintics in Euclidean and Minkowski planes. The case of Euclidean cubics was thoroughly treated in [10]. We give new proofs of some of the results of this paper and provide an analogical analysis for PH cubics in the Minkowski plane and planar PH and MPH quintics. Moreover, we introduce a novel approach to classifying PH curves using the so-called preimage of a transformation. This notion is closely related to the preimage curve of a $\mathrm{PH}$ curve. Based on this new approach, as the main result of the present paper, we give a classification of planar $\mathrm{PH}$ and MPH quintics and give simpler proofs of the classification of planar and spatial PH and MPH cubics, cf. [10, 11,23, 32].

The paper is organized as follows. In Section 2 we recall some basic facts about the Minkowski plane, Clifford algebras and PH curves. Section 3 introduces the preimage of a transformation, a notion which plays a key role in classifying PH curves. In Section 4 we study the control polygon properties and shapes of cubics in the Euclidean and Minkowski planes. In Section 5 we provide a similar analysis for Euclidean and Minkowski PH quintics. Finally we conclude the paper.

\section{Preliminaries}

In this section we summarize some fundamental results concerning PH curves in the Euclidean and Minkowski plane and their representation using complex numbers and Clifford algebra $\mathcal{C}(1,1)$.

\subsection{Minkowski plane}

The two-dimensional Minkowski space $\mathbb{R}^{1,1}$ is a real linear space with an indefinite inner product given by the matrix $G=\operatorname{diag}(1,-1)$. We use the upper index 1,1 to emphasize the signature of the inner product. Some authors use $\mathbb{R}^{2}$ or $\mathbb{R}^{2,1}$ to denote the Minkowski plane.

The inner product of two vectors $\mathbf{u}=\left(u_{1}, u_{2}\right)^{\top}, \mathbf{v}=\left(v_{1}, v_{2}\right)^{\top}, \mathbf{u}, \mathbf{v} \in \mathbb{R}^{1,1}$ is defined as

$$
\langle\mathbf{u}, \mathbf{v}\rangle=\mathbf{u}^{\top} G \mathbf{v}=u_{1} v_{1}-u_{2} v_{2} .
$$


Table 1. Multiplication table for $\mathcal{C}(1,1)$.

\begin{tabular}{|c||c|c|c|c|}
\hline$\cdot$ & $\mathbf{1}$ & $\mathbf{e}_{1}$ & $\mathbf{e}_{2}$ & $\mathbf{e}_{12}$ \\
\hline \hline $\mathbf{1}$ & $\mathbf{1}$ & $\mathbf{e}_{1}$ & $\mathbf{e}_{2}$ & $\mathbf{e}_{12}$ \\
\hline $\mathbf{e}_{1}$ & $\mathbf{e}_{1}$ & $\mathbf{1}$ & $\mathbf{e}_{12}$ & $\mathbf{e}_{2}$ \\
\hline $\mathbf{e}_{2}$ & $\mathbf{e}_{2}$ & $-\mathbf{e}_{12}$ & $-\mathbf{1}$ & $-\mathbf{e}_{1}$ \\
\hline $\mathbf{e}_{12}$ & $\mathbf{e}_{12}$ & $-\mathbf{e}_{2}$ & $\mathbf{e}_{1}$ & $\mathbf{1}$ \\
\hline
\end{tabular}

The two axes spanned by the vectors $\mathbf{e}_{1}=(1,0)^{\top}$ and $\mathbf{e}_{2}=(0,1)^{\top}$ will be denoted as the $x$ - and $y$-axis, respectively.

Since the quadratic form defined by $G$ is not positive definite as in the Euclidean case, the square norm of $\mathbf{u}$ defined by $\|\mathbf{u}\|^{2}=\langle\mathbf{u}, \mathbf{u}\rangle$ may be positive, negative or zero. Motivated by the theory of relativity one distinguishes three so-called "causal characters' of vectors. A vector $\mathbf{u}$ is said to be space-like if $\|\mathbf{u}\|^{2}>0$, time-like if $\|\mathbf{u}\|^{2}<0$, and light-like (or isotropic) if $\|\mathbf{u}\|^{2}=0$.

A linear mapping $L: \mathbb{R}^{1,1} \rightarrow \mathbb{R}^{1,1}$ is called a Lorentz transform or Minkowski rotation if it preserves the Minkowski inner product, i.e. $\langle\mathbf{u}, \mathbf{v}\rangle=\langle L \mathbf{u}, L \mathbf{v}\rangle$ for all $\mathbf{u}, \mathbf{v} \in \mathbb{R}^{1,1}$. The Lorentz transforms form the Lorentz group $\mathcal{L}=O(1,1)$.

Any Lorentz transform is described by a $2 \times 2$-matrix $L=\left(l_{i, j}\right)_{i, j=1,2}$. Its column vectors $\mathbf{l}_{1}$ and $\mathbf{l}_{2}$ satisfy $\left\langle\mathbf{l}_{i}, \mathbf{l}_{j}\right\rangle=G_{i, j}, i, j \in\{1,2\}$, i.e., they form an orthonormal basis of $\mathbb{R}^{1,1}$ with respect to the inner product (1). The equation $\left\langle\mathbf{l}_{2}, \mathbf{l}_{2}\right\rangle=G_{2,2}=-1$ implies $l_{22}^{2} \geq 1$. The Lorentz transform $L$ is said to be orthochronous if $l_{22} \geq 1$. Obviously, the determinant of any Lorentz transform $L$ equals to \pm 1 . The special ones are characterized by $\operatorname{det}(L)=1$.

The Lorentz group $\mathcal{L}$ consists of four components. The special orthochronous Lorentz transforms form the subgroup $S_{+}(1,1)$ which consists of matrices of the type

$$
\left(\begin{array}{c}
\cosh \alpha \sinh \alpha \\
\sinh \alpha \cosh \alpha
\end{array}\right)
$$

The remaining three components are $T_{1} S O_{+}(1,1), T_{2} S O_{+}(1,1)$ and $T_{1} T_{2} S O_{+}(1,1)$, where $T_{1}=\operatorname{diag}(1,-1)$ and $T_{2}=\operatorname{diag}(-1,1)$.

\subsection{The Clifford algebra $\mathcal{C}(1,1)$}

Any real linear space, which is equipped with a non-degenerate quadratic form, has an associated Clifford algebra, see [6,27] for a more detailed introduction. In particular we are interested in the Clifford algebra $\mathcal{C}(1,1)$, which is linked to the Minkowski plane $\mathbb{R}^{1,1}$.

This Clifford algebra has three different classes of basis elements: the scalar identity element $\mathbf{1}$, the orthonormal basis vectors $\mathbf{e}_{1}, \mathbf{e}_{2}$ and the pseudo-scalar $\mathbf{e}_{12}$. The noncommutative multiplication $\cdot$ can be deduced from the basic relations $\mathbf{e}_{1}^{2}=-\mathbf{e}_{2}^{2}=\mathbf{1}$ and $\mathbf{e}_{1} \cdot \mathbf{e}_{2}=\mathbf{e}_{2} \cdot \mathbf{e}_{1} \mathbf{e}_{12}$, see Table 1 . 
Any element of the Clifford algebra is a linear combination of these basis elements. In order to simplify the notation, we shall use vectors in $\mathbb{R}^{4}$ to represent them,

$$
A=\left[a_{0}, a_{1}, a_{2}, a_{3}\right]=a_{0} \mathbf{1}+a_{1} \mathbf{e}_{1}+a_{2} \mathbf{e}_{2}+a_{3} \mathbf{e}_{12} .
$$

The conjugation of elements and the squared norm are defined as

$$
\begin{aligned}
\bar{A} & =\left[a_{0},-a_{1},-a_{2},-a_{3}\right] \quad \text { and } \\
N(A) & =A \cdot \bar{A}=a_{0}^{2}-a_{1}^{2}+a_{2}^{2}-a_{3}^{2}
\end{aligned}
$$

respectively. The operation of conjugation satisfies $\overline{A \cdot B}=\bar{B} \cdot \bar{A}$.

All vectors of $\mathbb{R}^{1,1}$ will be identified with pure vectors

$$
\mathbf{c}=\left(c_{1}, c_{2}\right)^{\top}=c_{1} \mathbf{e}_{1}+c_{2} \mathbf{e}_{2}=\left[0, c_{1}, c_{2}, 0\right] \in \mathcal{C}(1,1) .
$$

The norms in $\mathbb{R}^{1,1}$ and $\mathcal{C}(1,1)$ are related by

$$
\|\mathbf{c}\|^{2}=-N(\mathbf{c}) .
$$

The set of scalars combined with pseudoscalars forms a subalgebra

$$
\mathbb{H}^{1,1}=\mathbb{R} \mathbf{1}+\mathbb{R} \mathbf{e}_{12}
$$

of the Clifford algebra $\mathcal{C}(1,1)$. Its elements will be represented by calligraphic characters, $\mathcal{A}=\left[a_{0}, 0,0, a_{3}\right]=a_{0} \mathbf{1}+a_{3} \mathbf{e}_{12}$.

Definition 1. We define on the algebra $\mathrm{C}(1,1)$ the commutative multiplication

$$
A \star B:=\frac{1}{2}\left(A \cdot \mathbf{e}_{1} \cdot \bar{B}+B \cdot \mathbf{e}_{1} \cdot \bar{A}\right) .
$$

Corresponding $n$-th powers will be denoted $A^{n \star}=\underbrace{A \star A \star \ldots \star A}_{n \star}$.

Note, that $\mathcal{A} \star \mathcal{B}$ is always a pure vector.

Now we state the following Lemmas, which we will make use of in the next section.

Lemma 1. Let $\mathbf{a}$ and $\mathbf{b}$ be two pure vectors. Then

$$
N(\mathbf{a} \star \mathbf{b})=-N(\mathbf{a}) N(\mathbf{b}) .
$$

Proof. By using the definitions of Clifford norm and Minkowski inner product, one can check that both sides of (3) are equal to $-\left(a_{1}^{2}-a_{2}^{2}\right)\left(b_{1}^{2}-b_{2}^{2}\right)$.

Lemma 2. Let $\mathbf{h}=\left[0, h_{1}, h_{2}, 0\right]$ be a pure space-like or light-like vector and $\mathcal{A}=$ $\left[a_{0}, 0,0, a_{3}\right] \in \mathbb{H}^{1,1}$. Then all solutions of the equation

$$
\mathbf{h}=\mathcal{A} \star \mathcal{A}
$$

are given by

$$
a_{0}= \pm \sqrt{\frac{h_{1} \pm \sqrt{h_{1}^{2}-h_{2}^{2}}}{2}}, \quad a_{3}=\mp \sqrt{\frac{h_{1} \mp \sqrt{h_{1}^{2}-h_{2}^{2}}}{2}} .
$$

Proof. By using the definition of the symmetric product, the equation (4) is equivalent to the system

$$
a_{0}^{2}+a_{3}^{2}=h_{1} \quad \text { and } \quad 2 a_{0} a_{3}=-h_{2} .
$$

Solving this system, which yields a biquadratic equation, concludes the proof. 


\subsection{Pythagorean hodograph curves}

Given any vector space $\mathbb{V}$ with (possibly indefinite) dot product we can define Pythagorean hodograph $(\mathrm{PH})$ curves, as polynomial curves in $\mathbb{V}$ with polynomial speed, i.e., as polynomial mappings $\mathbf{p}: \mathbb{R} \rightarrow \mathbb{V}$ such that $\left\|\mathbf{p}^{\prime}\right\|$ is a polynomial in $t$. Obviously, this definition is invariant with respect to the mappings preserving the dot product. In this paper we will analyse in detail the cases $\mathbb{V}=\mathbb{R}^{2}$ and $\mathbb{V}=\mathbb{R}^{1,1}$. However, many of our approaches can be applied in general.

\subsection{Planar Euclidean PH curves}

The hodograph of a planar curve $\mathbf{p}(t)=(x(t), y(t))^{\top}$ of degree $n$ is the curve $\mathbf{h}(t)=$ $\left(x^{\prime}(t), y^{\prime}(t)\right)^{\top}$ of degree $n-1$, where ' denotes the first derivative. For the remainder of the paper the hodograph of a curve $\mathbf{p}(t)$ will be denoted $\mathbf{h}(t)$.

Recall that a polynomial curve is called Pythagorean Hodograph $(P H)$, see [10] if the length of its tangent vector depends in a (piecewise) polynomial way on the parameter. More precisely, $\mathbf{p}(t)=(x(t), y(t))^{\top}$ is called a planar $P H$ curve if there exists a polynomial $\sigma(t)$ such that

$$
x^{\prime}(t)^{2}+y^{\prime}(t)^{2}=\sigma^{2}(t) .
$$

Following the classical result of [26] the general solution of (5) takes the form

$$
\begin{aligned}
x^{\prime}(t) & =q(t)\left(u^{2}(t)-v^{2}(t)\right), \\
y^{\prime}(t) & =q(t)(2 u(t) v(t)), \\
\sigma(t) & =q(t)\left(u^{2}(t)+v^{2}(t)\right) .
\end{aligned}
$$

If all real roots of $\operatorname{gcd}\left(x^{\prime}(t), y^{\prime}(t)\right)$ have even multiplicity ${ }^{3}$, then $q(t)$ can be omitted. In the sequel we will suppose this case, since the common factor $q(t)$ raises the degree of the curve without yielding more geometric flexibility (it does not modify tangent directions).

Any $\mathrm{PH}$ curve can be therefore represented through its preimage

$$
\mathcal{A}(t)=(u(t), v(t)) .
$$

We will call the two dimensional vector space of $u$ and $v$ the representation space and denote it $S_{\mathbb{R}^{2}}$. Then we can describe the whole situation by the following diagram

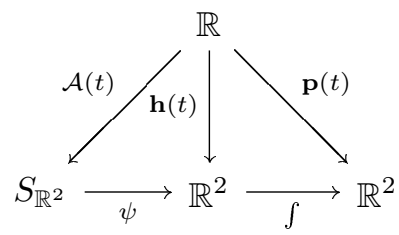

where $\psi:(u, v)^{\top} \rightarrow\left(u^{2}-v^{2}, 2 u v\right)^{\top}$ will be called representation mapping.

The representation result can be reformulated using complex numbers. Any planar curve $\mathbf{p}(t)=(x(t), y(t))^{\top}$ is identified with the complex function $\mathbf{p}(t)=x(t)+y(t) \mathbf{i}$

\footnotetext{
${ }^{3}$ This includes the generic case $\operatorname{gcd}\left(x^{\prime}(t), y^{\prime}(t)\right)=1$.
} 
and any preimage $\mathcal{A}=(u(t), v(t))^{\top}$ with the complex function $\mathcal{A}(t)=u(t)+v(t)$ i. Then the characterization mapping $\psi$ corresponds to complex squaring, since $\mathcal{A}^{2}=$ $(u+v \mathbf{i})^{2}=\left(u^{2}-v^{2}\right)+2 u v \mathbf{i}$. The relation between the hodograph of a PH curve and its preimage thus becomes

$$
\mathbf{h}(t)=\mathcal{A}(t)^{2} .
$$

\subsection{Minkowski Pythagorean hodograph curves}

Following [29], Minkowski Pythagorean Hodograph (MPH) curves are defined similarly to PH curves, but with respect to the norm induced by the Minkowski inner product. More precisely, a polynomial curve $\mathbf{p} \in \mathbb{R}^{1,1}, \mathbf{p}(t)=(x(t), y(t))^{\top}$ is called an MPH curve if

$$
\|\mathbf{h}(t)\|^{2}={x^{\prime}}^{2}(t)-y^{\prime 2}(t)=\sigma^{2}(t)
$$

for some polynomial $\sigma$.

Due to the definition of MPH curves, the tangent vector $\mathbf{p}^{\prime}(t)$ cannot be time-like. Also, light-like tangent vectors correspond to roots of the polynomial $\sigma(t)$ in (6). Each regular point of an MPH curve has a space-like or light-like tangent vector.

The equation (6) holds if and only if there exist polynomials $u(t), v(t), q(t)$ such that

$$
\begin{aligned}
x^{\prime}(t) & =q(t)\left(u^{2}(t)+v^{2}(t)\right), \\
y^{\prime}(t) & =q(t)(2 u(t) v(t)), \\
\sigma(t) & =q(t)\left(u^{2}(t)-v^{2}(t)\right) .
\end{aligned}
$$

Again we will suppose that $q(t)=1$, in which case the following representation diagram holds

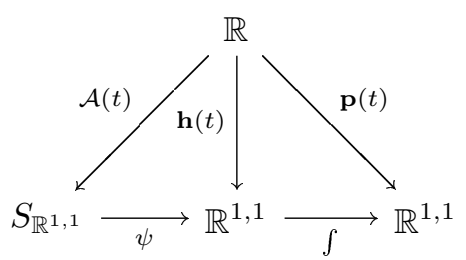

where the representation mapping is $\psi:(u, v)^{\top} \rightarrow\left(u^{2}+v^{2}, 2 u v\right)^{\top}$.

The representation result can be reformulated using Clifford algebra $\mathcal{C}(1,1)$, see [6], by identifying vectors with elements of the algebra, cf. (2). Any planar curve $\mathbf{p}(t)=$ $(x(t), y(t))^{\top}$ is identified with the function $\mathbf{p}(t)=x(t) \mathbf{e}_{1}+y(t) \mathbf{e}_{2}$ and any preimage $\mathcal{A}=(u(t), v(t))^{\top}$ with the function in the subalgebra $\mathbb{H}^{1,1}$ by setting $\mathcal{A}(t)=u(t)+$ $v(t) \mathbf{e}_{12}$. Then the characterization mapping $\psi$ corresponds to $\mathcal{A}(t) \cdot \mathbf{e}_{1} \cdot \overline{\mathcal{A}}(t)$ and the relation between a PH curve and its preimage thus becomes

$$
\mathbf{h}(t)=\mathcal{A}(t) \cdot \mathbf{e}_{1} \cdot \overline{\mathcal{A}}(t)=\mathcal{A}(t)^{2 \star} .
$$

\section{Inducing transformations via preimages}

In the sequel we want to classify low degree PH curves up to "similarities". More precisely, we want to classify PH curves in $\mathbb{V}$ with respect to the action of the conformal 
group $C O(\mathbb{V})=O N(\mathbb{V}) \times \mathbb{R}_{+}$, where $O N(\mathbb{V})$ is the group of all transformations preserving the inner product. Since the PH curves are represented by preimages in the representation space, we will try to realize the classification already on the level of preimages. In this chapter we will investigate which transformations of the representation space produce transformations of the space $\mathbb{V}$ belonging to $C O(\mathbb{V})$.

\subsection{General concept}

As we have seen in Section 2, the general representation scheme for PH curves is

$$
S_{\mathbb{V}} \stackrel{\psi}{\longrightarrow} \mathbb{V} \stackrel{\rho}{\longrightarrow} \mathbb{V} .
$$

The integration commutes with any affine transformation of the hodograph and from the transformation point of view it only can produce a translation through a choice of integration constants. We are looking for certain preimages of conformal mappings, i.e., for linear mappings of $S_{\mathbb{V}}$ which produce conformal mappings of $\mathbb{V}$. This concept is formalized in the following definition.

Definition 2. An element of $\lambda \in G L\left(S_{\mathbb{V}}\right)$ will be called the conformal preimage of $\mu \in C O(\mathbb{V})$ if and only if $\psi(\lambda(a))=\mu(\psi(a))$ for any element $a \in S_{\mathbb{V}}$.

In other words, the following commutative diagram holds

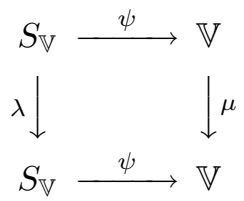

Indeed, one $\mu$ can have several preimages. On the other hand, due to the surjectivity of $\psi$ any $\lambda$ can be the preimage of at most one $\mu$.

Lemma 3. The set of all preimages of all $\mu \in C O(\mathbb{V})$ forms a subgroup of $G L\left(S_{\mathbb{V}}\right)$, which will be called the preimage group and denoted $P C O\left(S_{\mathbb{V}}\right)$.

Proof. It is a straightforward observation that $\mathbf{1}_{S_{\mathbb{V}}}$ is the preimage of $\mathbf{1}_{\mathbb{V}}$. Moreover, if $\lambda$ is the preimage of $\mu$ and $\lambda^{\prime}$ is the preimage of $\mu^{\prime}$ then $\lambda^{-1}$ is the preimage of $\mu^{-1}$ and $\lambda\left(\lambda^{\prime}\right)$ is the preimage of $\mu\left(\mu^{\prime}\right)$.

Before we turn our attention to concrete examples of $\mathbb{V}$, we consider the influence of the linear reparameterization of the preimage on the shape of the final PH curve. Indeed, reparameterization is (besides the application of a transformation) another natural way how to modify the preimage. The following lemma follows directly from the integration by substitution rule.

Lemma 4. Let $\mathcal{A}(t): \mathbb{R} \rightarrow S_{\mathbb{V}}$ be a polynomial preimage of the PH curve

$$
\mathbf{p}(t)=\int \psi(\mathcal{A}(t)): \mathbb{R} \rightarrow \mathbb{V}
$$

and $k \neq 0, l$ real constants. Then $\mathcal{A}(k t+l)$ is the preimage of the curve $\frac{1}{k} \mathbf{p}(k t+l)$. Therefore, reparameterizing the preimage corresponds to scaling $\mathbf{p}(t)$. 


\subsection{Group $\mathrm{PCO}\left(S_{\mathbb{R}^{2}}\right)$}

Recall that in the case of Euclidean planar PH curves, the representation mapping has the form

$$
\psi:(u, v)^{\top} \rightarrow\left(u^{2}-v^{2}, 2 u v\right)^{\top}
$$

The conformal group $C O\left(\mathbb{R}^{2}\right)$ is generated by mappings of the form $\left(\begin{array}{rr}a & b \\ -b & a\end{array}\right)$ together with the mapping given by $\left(\begin{array}{rr}1 & 0 \\ 0 & -1\end{array}\right)$. An element $\left(\begin{array}{cc}k & l \\ m & n\end{array}\right) \in G L\left(S_{\mathbb{R}^{2}}\right)$ is the preimage of $\left(\begin{array}{rr}a & b \\ -b & a\end{array}\right) \in C O\left(\mathbb{R}^{2}\right)$ if and only if for any $(u, v)^{\top} \in S_{\mathbb{R}^{2}}$ it holds

$$
\left(\begin{array}{rr}
a & b \\
-b & a
\end{array}\right)\left(\begin{array}{c}
u^{2}-v^{2} \\
2 u v
\end{array}\right)=\left(\begin{array}{c}
(k u+l v)^{2}-(m u+n v)^{2} \\
2(k u+l v)(m u+n v)
\end{array}\right) .
$$

Comparing the coefficients we obtain the following system of equations

$$
\begin{aligned}
& a=k^{2}-m^{2}=n^{2}-l^{2}=k n+l m, \\
& b=k l-m n=-2 k m=2 l n,
\end{aligned}
$$

which implies $n=k, m=-l, a=k^{2}-l^{2}, b=2 k l$. These equations are well known in the context of PH curves and it has a solution for any $a, b$. Similarly,

$$
\left(\begin{array}{rr}
1 & 0 \\
0 & -1
\end{array}\right),\left(\begin{array}{rr}
-1 & 0 \\
0 & 1
\end{array}\right) \in G L\left(S_{\mathbb{R}^{2}}\right)
$$

are the two preimages of $\left(\begin{array}{rr}1 & 0 \\ 0 & -1\end{array}\right) \in G L\left(\mathbb{R}^{2}\right)$. Summing up, we obtain the following result.

Theorem 1. The preimage group $P C O\left(S_{\mathbb{R}^{2}}\right)$ is the conformal group $C O\left(\mathbb{R}^{2}\right)$ and any element of $C O\left(\mathbb{R}^{2}\right)$ has its preimage in $P C O\left(S_{\mathbb{R}^{2}}\right)$.

\subsection{Group $\operatorname{PCO}\left(S_{\mathbb{R}^{1,1}}\right)$}

Recall that in the case of Minkowski planar PH curves, the representation mapping has the form

$$
\psi:(u, v)^{\top} \rightarrow\left(u^{2}+v^{2}, 2 u v\right)^{\top}
$$

The conformal group $C O\left(\mathbb{R}^{1,1}\right)$ is generated by mappings of the form $\left(\begin{array}{ll}a & b \\ b & a\end{array}\right)$, where

$$
|a|>|b|, a>0
$$

together with the mappings given by $\left(\begin{array}{rr}1 & 0 \\ 0 & -1\end{array}\right)$ and $\left(\begin{array}{rr}-1 & 0 \\ 0 & 1\end{array}\right)$. 


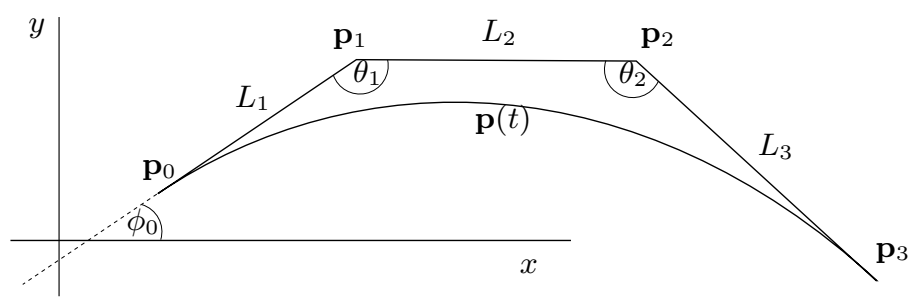

Fig. 1. Planar cubic and its control polygon.

An element $\left(\begin{array}{cc}k & l \\ m & n\end{array}\right) \in G L\left(S_{\mathbb{R}^{1,1}}\right)$ is the preimage of $\left(\begin{array}{ll}a & b \\ b & a\end{array}\right) \in C O\left(\mathbb{R}^{1,1}\right)$ if and only if for any $(u, v)^{\top} \in S_{\mathbb{R}^{1,1}}$ it holds

$$
\left(\begin{array}{ll}
a & b \\
b & a
\end{array}\right)\left(\begin{array}{c}
u^{2}+v^{2} \\
2 u v
\end{array}\right)=\left(\begin{array}{c}
(k u+l v)^{2}+(m u+n v)^{2} \\
2(k u+l v)(m u+n v)
\end{array}\right) .
$$

Comparing the coefficients we obtain the following system of equations

$$
\begin{aligned}
& a=k^{2}+m^{2}=n^{2}+l^{2}=k n+l m, \\
& b=k l+m n=2 k m=2 l n,
\end{aligned}
$$

which implies $n=k, m=l, a=k^{2}+l^{2}, b=2 k l$. Again, when (7) hold, this system has always a solution. Moreover, $\left(\begin{array}{rr}1 & 0 \\ 0 & -1\end{array}\right),\left(\begin{array}{rr}-1 & 0 \\ 0 & 1\end{array}\right) \in G L\left(S_{\mathbb{R}^{1,1}}\right)$ are the two preimages of $\left(\begin{array}{rr}1 & 0 \\ 0 & -1\end{array}\right) \in G L\left(\mathbb{R}^{1,1}\right)$. On the other hand, the element $\left(\begin{array}{rr}-1 & 0 \\ 0 & 1\end{array}\right) \in$ $G L\left(\mathbb{R}^{1,1}\right)$ has no preimage. Summing up, we arrive at the following result

Theorem 2. The preimage group $P C O\left(S_{\mathbb{R}^{1,1}}\right)$ is generated by the elements of the form $\left(\begin{array}{ll}k & l \\ l & k\end{array}\right)$, where $k \neq l$ can be arbitrary and by the elements $\left(\begin{array}{rr}1 & 0 \\ 0 & -1\end{array}\right),\left(\begin{array}{rr}-1 & 0 \\ 0 & 1\end{array}\right)$. A preimage exists for an element of $C O\left(\mathbb{R}^{1,1}\right)$ provided that its first matrix element is positive.

\section{Planar PH and MPH cubics}

\subsection{Euclidean PH cubics}

We start with recalling the properties of the control polygons of planar PH cubics, cf. [10]. Let $\mathbf{p}(t)$ be a planar cubic in $\mathbb{R}^{2}$ expressed in the Bernstein-Bézier representation [19]

$$
\mathbf{p}(t)=\sum_{i=0}^{3} \mathbf{p}_{i} B_{i}^{3}(t), \quad t \in[0,1]
$$


where $\mathbf{p}_{i}$, are the control points and $B_{j}^{n}(t)=\left(\begin{array}{c}n \\ j\end{array}\right) t^{j}(1-t)^{n-j}$ are the Bernstein polynomials. Moreover, let $L_{1}, L_{2}$ and $L_{3}$ be the lengths of the control polygon legs and $\theta_{1}$, $\theta_{2}$ be the control polygon angles included at the interior vertices $\mathbf{p}_{1}, \mathbf{p}_{2}$, respectively see Fig. 1. Then, as shown in [10], Section 4, the following theorem holds.

Theorem 3 (Farouki 1990). Let $\mathbf{p}(t)$ be a cubic in $\mathbb{R}^{2}$. Then $\mathbf{p}(t)$ is a planar PH cubic if and only if

$$
L_{2}^{2}=L_{1} L_{3} \quad \text { and } \quad \theta_{1}=\theta_{2}
$$

Using the PH representation map (see [6]) and the complex numbers setting we reformulate this theorem and provide an alternative proof. We express the hodograph $\mathbf{h}(t)$ of $\mathbf{p}(t)$ and its preimage $\mathcal{A}(t)$ in the Bernstein-Bézier representation

$$
\mathbf{h}(t)=\sum_{i=0}^{2} \mathbf{h}_{i} B_{i}^{2}(t), \quad \mathcal{A}(t)=\sum_{i=0}^{1} \mathcal{A}_{i} B_{i}^{1}(t), \quad t \in[0,1],
$$

where $\mathbf{h}_{i}$ and $\mathcal{A}_{i}$ are the complex control points.

Theorem 4. Let $\mathbf{p}(t)$ be a cubic in $\mathbb{R}^{2}$. Then $\mathbf{p}(t)$ is a planar PH cubic if and only if

$$
\mathbf{h}_{0} \mathbf{h}_{2}=\mathbf{h}_{1}^{2} \text {. }
$$

Proof. The relation between the hodograph and the preimage

$$
\mathbf{h}(t)=\mathcal{A}(t)^{2}
$$

can be expressed using the control points as

$$
\begin{aligned}
& \mathbf{h}_{0}=\mathcal{A}_{0}^{2}, \\
& \mathbf{h}_{1}=\mathcal{A}_{0} \mathcal{A}_{1}, \\
& \mathbf{h}_{2}=\mathcal{A}_{1}^{2} .
\end{aligned}
$$

From the basic properties of complex numbers it follows that

$$
\mathbf{h}_{0} \mathbf{h}_{2}=\mathbf{h}_{1}^{2} .
$$

Now for the converse statement. Let $\mathbf{p}(t)$ be a planar cubic with its hodograph control points $\mathbf{h}_{i}, i=0,1,2$ such that it satisfies (11). First, compute $\mathcal{A}_{0}$ from the first equation in (10) using any complex square root. Then, compute $\mathcal{A}_{1}$ from $\mathbf{h}_{1}=\mathcal{A}_{0} \mathcal{A}_{1}$. Finally, one can see that the last equation in (10) holds. Therefore, $\mathcal{A}_{0}$ and $\mathcal{A}_{1}$ form preimage control points for $\mathbf{p}(t)$. Hence, $\mathbf{p}(t)$ is a planar $\mathrm{PH}$ cubic.

Remark 1. Let $\phi_{i}$ be the argument of $\mathbf{h}_{i}, i=0,1,2$, in its polar form. Then (11) is equivalent to the system

$$
\begin{aligned}
\left|\mathbf{h}_{0}\right|\left|\mathbf{h}_{2}\right| & =\left|\mathbf{h}_{1}\right|^{2}, \\
\phi_{0}+\phi_{2} & =2 \phi_{1},
\end{aligned}
$$

where $\left|\mathbf{h}_{i}\right|$ denotes the absolute value of the complex number $\mathbf{h}_{i}$. Moreover, $L_{i}=$ $\frac{1}{3}\left|\mathbf{h}_{i-1}\right|$ for $i=1,2,3$ and $\theta_{1}, \theta_{2}$ are the control polygon angles included at the interior vertices $\mathbf{p}_{1}, \mathbf{p}_{2}$, respectively. Thus one can see that (9) is equivalent to the system (8). 

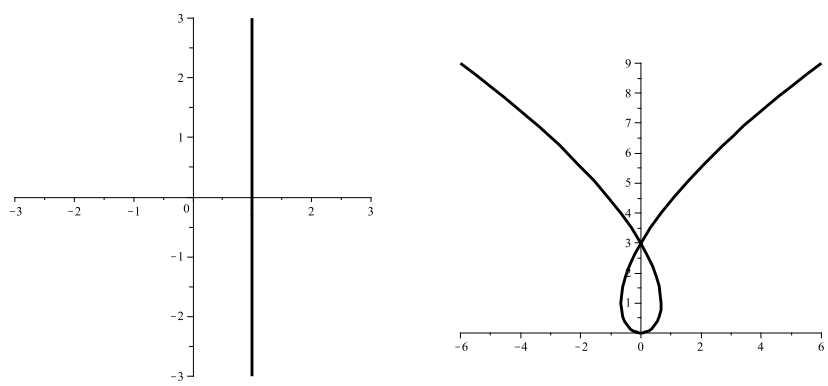

Fig. 2. The "unique" Euclidean PH cubic - the Tschirnhausen cubic (right) and its preimage (left).

Now, using the tools of Section 3, we give a new proof of the classification result for Euclidean PH cubics - see [10] for the original proof.

Theorem 5. Up to Euclidean similarities there is only one Euclidean PH planar cubic, see Fig. 2. More precisely, any planar Euclidean PH cubic can be transformed by some element of $C O\left(\mathbb{R}^{2}\right)$ and by a reparameterization to the Tschirnhausen cubic

$$
\left(t-\frac{t^{3}}{3}, t^{2}\right)^{\top}
$$

Proof. It follows from Theorem 1 that Euclidean similarities of PH cubics can be generated by Euclidean similarities of their preimages. The preimage of any $\mathrm{PH}$ cubic $\mathbf{p}$ is some straight line $l$. We can assume that $l$ does not pass through the origin, since if it does, the resulting curve is a straight line. By a suitable conformal transformation $\lambda$ (see Definition 2) it can be mapped onto the line passing through the point $(1,0)$ and parallel to the $y$ axis and then reparameterized so that it becomes $(1, t)^{\top}$, which is the preimage of the cubic (12). It follows from Theorem 1 and Lemma 4 that $\lambda$ and the reparameterization of the preimage will produce a similarity which together with the reparameterization transform $\mathbf{p}$ to the desired form (12).

\subsection{MPH cubics}

Now, we move on to planar MPH cubics. Using the PH representation map (see [6]) and the Clifford algebra $\mathcal{C}(1,1)$ setting we express planar MPH cubics $\mathbf{p}(t)$, their hodographs $\mathbf{h}(t)$ and the preimages $\mathcal{A}(t)$ as

$$
\mathbf{p}(t)=\sum_{i=0}^{3} \mathbf{p}_{i} B_{i}^{3}(t), \quad \mathbf{h}(t)=\sum_{i=0}^{2} \mathbf{h}_{i} B_{i}^{2}(t), \quad \mathcal{A}(t)=\sum_{i=0}^{1} \mathcal{A}_{i} B_{i}^{1}(t), \quad t \in[0,1],
$$

where $\mathbf{p}_{i}, \mathbf{h}_{i}$ and $\mathcal{A}_{i}$ are the control points. In this setting, $\mathbf{p}(t)$ and its hodograph are described by pure vectors, whereas the preimage lies in the subalgebra $\mathbb{H}^{1,1}$. The following Theorem is an analogy to the Euclidean case. 
Theorem 6. A planar cubic curve $\mathbf{p}(t)$ in $\mathbb{R}^{1,1}$ is an MPH curve if and only if its hodograph satisfies

$$
\mathbf{h}_{0} \star \mathbf{h}_{2}=\mathbf{h}_{1} \star \mathbf{h}_{1} .
$$

Proof. The relation between the hodograph and the preimage

$$
\mathbf{h}(t)=\mathcal{A}(t) \star \mathcal{A}(t)
$$

can be expressed using the control points as

$$
\begin{aligned}
& \mathbf{h}_{0}=\mathcal{A}_{0} \star \mathcal{A}_{0}, \\
& \mathbf{h}_{1}=\mathcal{A}_{0} \star \mathcal{A}_{1}, \\
& \mathbf{h}_{2}=\mathcal{A}_{1} \star \mathcal{A}_{1} .
\end{aligned}
$$

Exploiting the commutativity and associativity of the symmetric product $\star$ on pure vectors, one can see that (14) yields (13).

In order to prove the converse statement, we need to distinguish two cases, since light-like elements in $\mathcal{C}(1,1)$ have no inverse elements. Let $\mathbf{p}(t)$ be a planar cubic with its hodograph control points $\mathbf{h}_{i}, i=0,1,2$ such that it satisfies (13).

First, assume that both $\mathbf{h}_{0}$ and $\mathbf{h}_{2}$ are light-like. Then, from (13) and Lemma 1 it follows that $\mathbf{h}_{1}$ is light-like as well. Therefore, if $\mathcal{A}_{0}$ and $\mathcal{A}_{1}$ exist, they must be lightlike too and can be written as

$$
\mathcal{A}_{0}=\left[a_{0}, 0,0, \pm a_{0}\right], \quad \mathcal{A}_{1}=\left[a_{1}, 0,0, \pm a_{1}\right] .
$$

Checking different cases with respect to the 4 sign combinations, one can see that this preimage either gives a light-like straight line as the corresponding MPH curve, or the preimage gives $\mathbf{h}_{1}=[0,0,0,0]$. In the latter case one can compute the preimage from the first and third equation of (14) using Lemma 2, thus proving that $\mathbf{p}(t)$ is an MPH cubic.

Second, let $\mathbf{h}_{0}$ be space-like (if $\mathbf{h}_{0}$ is light-like we simple interchange the roles of $\mathbf{h}_{0}$ and $\mathbf{h}_{2}$ ). Then, as in the Euclidean case, we compute $\mathcal{A}_{0}$ from the first equation of (14) using any solution, see Lemma 2. Since $N\left(\mathbf{h}_{0}\right)=N\left(\mathcal{A}_{0} \star \mathcal{A}_{0}\right)=-N\left(\mathcal{A}_{0}\right)^{2}$, we have that $N\left(\mathcal{A}_{0}\right) \neq 0$ and $\mathcal{A}_{0}$ is invertible. Therefore, using the second equation of (14), we can compute

$$
\mathcal{A}_{1}=\frac{\mathbf{h}_{1} \cdot \mathcal{A}_{0} \cdot \mathbf{e}_{1}}{N\left(\mathcal{A}_{0}\right)} .
$$

Indeed,

$$
\begin{aligned}
& \frac{\mathbf{h}_{1} \cdot \mathcal{A}_{0} \cdot \mathbf{e}_{1}}{N\left(\mathcal{A}_{0}\right)} \star \mathcal{A}_{0}=\frac{1}{2 N\left(\mathcal{A}_{0}\right)}\left(\mathbf{h}_{1} \cdot \mathcal{A}_{0} \cdot \mathbf{e}_{1} \cdot \mathbf{e}_{1} \cdot \overline{\mathcal{A}}_{0}+\mathcal{A}_{0} \cdot \mathbf{e}_{1} \cdot \overline{\mathbf{e}}_{1} \cdot \overline{\mathcal{A}}_{0} \cdot \overline{\mathbf{h}}_{1} \cdot\right)= \\
& \frac{1}{2 N\left(\mathcal{A}_{0}\right)}\left(\mathbf{h}_{1} \cdot \mathcal{A}_{0} \cdot \overline{\mathcal{A}}_{0}-\mathcal{A}_{0} \cdot \overline{\mathcal{A}}_{0} \cdot \overline{\mathbf{h}}_{1}\right)=\frac{1}{2}\left(\mathbf{h}_{1}-\overline{\mathbf{h}}_{1}\right)=\mathbf{h}_{1} .
\end{aligned}
$$

Now, we express

$$
\begin{aligned}
& \mathcal{A}_{1} \star \mathcal{A}_{1}=\frac{1}{N\left(\mathcal{A}_{0}\right)^{2}}\left(\mathbf{h}_{1} \cdot \mathcal{A}_{0} \cdot \mathbf{e}_{1} \cdot \mathbf{e}_{1} \cdot \overline{\mathbf{e}}_{1} \cdot \overline{\mathcal{A}}_{0} \cdot \overline{\mathbf{h}}_{1}\right)= \\
& -\frac{1}{N\left(\mathcal{A}_{0}\right)^{2}}\left(\mathbf{h}_{1} \cdot \mathcal{A}_{0} \cdot \mathbf{e}_{1} \cdot \overline{\mathcal{A}}_{0} \cdot \overline{\mathbf{h}}_{1}\right)=-\frac{1}{N\left(\mathcal{A}_{0}\right)^{2}}\left(\mathbf{h}_{1} \cdot\left(\mathcal{A}_{0} \star \mathcal{A}_{0}\right) \cdot \overline{\mathbf{h}}_{1}\right)= \\
& \frac{1}{N\left(h_{0}\right)}\left(\mathbf{h}_{1} \cdot \mathbf{h}_{0} \cdot \overline{\mathbf{h}}_{1}\right)
\end{aligned}
$$


and verify that

$$
\begin{aligned}
& \mathbf{h}_{0} \star\left(\mathcal{A}_{1} \star \mathcal{A}_{1}\right)=\mathbf{h}_{0} \star \frac{\mathbf{h}_{1} \cdot \mathbf{h}_{0} \cdot \overline{\mathbf{h}}_{1}}{N\left(\mathbf{h}_{0}\right)}=\frac{\left(\mathbf{h}_{0} \cdot \mathbf{e}_{1} \cdot \mathbf{h}_{1} \cdot \overline{\mathbf{h}}_{0} \cdot \overline{\mathbf{h}}_{1}+\mathbf{h}_{1} \cdot \mathbf{h}_{0} \cdot \overline{\mathbf{h}}_{1} \cdot \mathbf{e}_{1} \cdot \overline{\mathbf{h}}_{0}\right)}{2 N\left(\mathbf{h}_{0}\right)}= \\
& \frac{1}{2}\left(\mathbf{h}_{1} \cdot \mathbf{e}_{1} \cdot \overline{\mathbf{h}}_{1}+\mathbf{h}_{1} \cdot \mathbf{e}_{1} \cdot \overline{\mathbf{h}}_{1}\right)=\mathbf{h}_{1} \star \mathbf{h}_{1} .
\end{aligned}
$$

We assumed that

$$
\mathbf{h}_{0} \star \mathbf{h}_{2}=\mathbf{h}_{1} \star \mathbf{h}_{1}
$$

therefore $\mathcal{A}_{1} \star \mathcal{A}_{1}=\mathbf{h}_{2}$ as $\mathbf{h}_{0}$ is invertible. This completes the proof, since $\mathcal{A}_{0}$ and $\mathcal{A}_{1}$ give a preimage of $\mathbf{p}(t)$.

Still, we would like to obtain a geometric description of planar MPH curves as in the Euclidean case, see Theorem 3. However, as the Minkowski inner product is not positive definite, there is no straightforward definition of angle in the whole Minkowski plane. Nevertheless, the following Theorem holds.

Theorem 7. Let $\mathbf{p}(t)$ in $\mathbb{R}^{1,1}$ be a planar MPH cubic. Then

$$
\left\|\mathbf{h}_{0}\right\|\left\|\mathbf{h}_{2}\right\|=\left\|\mathbf{h}_{1}\right\|^{2}
$$

and

$$
\left\|\mathbf{h}_{2}\right\|^{2}\left\langle\mathbf{h}_{0}, \mathbf{h}_{1}\right\rangle^{2}=\left\|\mathbf{h}_{0}\right\|^{2}\left\langle\mathbf{h}_{1}, \mathbf{h}_{2}\right\rangle^{2} .
$$

Proof. Comparing Clifford norms of both sides of equation (13) gives

$$
N\left(\mathbf{h}_{0} \star \mathbf{h}_{2}\right)=N\left(\mathbf{h}_{1} \star \mathbf{h}_{1}\right) .
$$

As $\mathbf{h}_{0}, \mathbf{h}_{1}$ and $\mathbf{h}_{2}$ are pure vectors, applying Lemma 1 yields

$$
N\left(\mathbf{h}_{0}\right) N\left(\mathbf{h}_{2}\right)=N\left(\mathbf{h}_{1}\right)^{2} .
$$

As no leg of the control polygon of an MPH curve can be time-like, we arrive at (16).

Now, let $\mathcal{A}_{0}=\left[a_{0}, 0,0, a_{1}\right]$ and $\mathcal{A}_{1}=\left[a_{2}, 0,0, a_{3}\right]$ be the preimage control points of $\mathbf{p}(t)$. Using (14), one can verify that both sides of equation (17) are equal to

$$
-\left(a_{1} a_{3}-a_{2} a_{0}\right)^{2} N\left(\mathcal{A}_{0}\right)^{2} N\left(\mathcal{A}_{1}\right)^{2} \text {. }
$$

Remark 2. Let $L_{1}, L_{2}$ and $L_{3}$ be the lengths of the control polygon legs of $\mathbf{p}(t)$ computed with respect to the Minkowski inner product, i.e. $L_{i}=\frac{1}{3}\left\|\mathbf{h}_{i}\right\|$. We obtained an analogous condition for the lengths of the control polygon legs of an MPH curve as in the Euclidean case (cf. (8))

$$
L_{2}^{2}=L_{1} L_{3}
$$

In the Euclidean case, the two angles included between $h_{0}$ and $h_{1}, h_{1}$ and $h_{2}$ are the same, see Theorem 3. Using Euclidean inner product and norm, denoted by ${ }_{E}$, one can express this condition as

$$
\left\|\mathbf{h}_{2}\right\|_{E}\left\langle\mathbf{h}_{0}, \mathbf{h}_{1}\right\rangle_{E}=\left\|\mathbf{h}_{0}\right\|_{E}\left\langle\mathbf{h}_{1}, \mathbf{h}_{2}\right\rangle_{E}
$$

Consequently, equation (17) represents an analogy to the angular formula in Theorem 3. 
These observations lead us to the following definitions.

Definition 3. Let $\mathbf{h}=\left[0, h_{1}, h_{2}, 0\right]$ be a pure space-like vector. If $h_{1}>0$ then $\mathbf{h}$ is called a positive space-like vector.

Using basic geometry, one can see that any positive space-like vector can be represented using its Minkowski norm $\|\mathbf{h}\|$ and hyperbolic angle as

$$
\mathbf{h}=[0,\|\mathbf{h}\| \cosh \phi,\|\mathbf{h}\| \sinh \phi, 0] \text {. }
$$

Moreover, any positive space-like vector can be uniquely represented using its Minkowski norm and hyperbolic angle.

Definition 4. Let $\mathbf{a}$ and $\mathbf{b}$ be two positive space-like vectors. Then we define the angle $\theta$ included between $\mathbf{a}$ and $\mathbf{b}$ as

$$
\cosh \theta=\frac{\langle\mathbf{a}, \mathbf{b}\rangle}{\|\mathbf{a}\|\|\mathbf{b}\|} .
$$

Note that this definition of angle is invariant with respect to special Lorentz transforms. Moreover, the hyperbolic angle of a can be defined as the angle included between a and $\mathbf{e}_{1}$.

Lemma 5. Let $\mathbf{a}$ and $\mathbf{b}$ be two positive space-like vectors

$$
\mathbf{a}=[0,\|\mathbf{a}\| \cosh \phi,\|\mathbf{a}\| \sinh \phi, 0], \quad \mathbf{b}=[0,\|\mathbf{b}\| \cosh \psi,\|\mathbf{b}\| \sinh \psi, 0] .
$$

Then

$$
\mathbf{a} \star \mathbf{b}=-[0,\|\mathbf{a} \mid\| \mathbf{b}\|\cosh (\phi+\psi),\| \mathbf{a}\|\| \mathbf{b} \| \sinh (\phi+\psi), 0] .
$$

Proof. The claim is a direct consequence of the definition of the symmetric product $\star$ and the hyperbolic functions formulas

$$
\begin{aligned}
& \cosh (\phi+\psi)=\cosh \phi \cosh \psi+\sinh \phi \sinh \psi, \\
& \sinh (\phi+\psi)=\sinh \phi \cosh \psi+\cosh \phi \sinh \psi
\end{aligned}
$$

Now, let us assume that $\mathbf{h}_{0}, \mathbf{h}_{1}$ and $\mathbf{h}_{2}$ are positive space-like vectors with hyperbolic angles $\phi_{0}, \phi_{1}$ and $\phi_{2}$, respectively. Then Lemma 5 yields that both $-\mathbf{h}_{0} \star \mathbf{h}_{2}$ and $-\mathbf{h}_{1} \star \mathbf{h}_{1}$ are positive space-like vectors as well. Thus, we see that the equation

$$
\mathbf{h}_{0} \star \mathbf{h}_{2}=\mathbf{h}_{1} \star \mathbf{h}_{1}
$$

is equivalent to the system

$$
\begin{aligned}
& L_{2}^{2}=L_{1} L_{3}, \\
& \phi_{0}+\phi_{2}=2 \phi_{1} .
\end{aligned}
$$

Finally, let $\theta_{1}, \theta_{2}$ be the angles included between $\mathbf{h}_{0}$ and $\mathbf{h}_{1}, \mathbf{h}_{1}$ and $\mathbf{h}_{2}$, respectively. Collecting our results, we arrive at 
Theorem 8. Let $\mathbf{p}(t)$ be a cubic in $\mathbb{R}^{1,1}$ such that its hodograph control points are positive space-like vectors. Then $\mathbf{p}(t)$ is a planar MPH cubic if and only if

$$
L_{2}^{2}=L_{1} L_{3} \quad \text { and } \quad \theta_{1}=\theta_{2} .
$$

Proof. It only remains to show that $\theta_{1}=\theta_{2}$ is equivalent to $\phi_{0}+\phi_{2}=2 \phi_{1}$. We leave this computation as an exercise on inverse hyperbolic functions using the formulas

$$
\begin{aligned}
& \operatorname{arccosh}(x)=\ln \left(x+\sqrt{x^{2}-1}\right), \\
& \operatorname{arccosh}(x)+\operatorname{arccosh}(y)=\operatorname{arccosh}\left(x y+\sqrt{\left(x^{2}-1\right)\left(y^{2}-1\right)}\right) .
\end{aligned}
$$

Now, we will give a classification of Minkowski PH planar cubics.

Theorem 9. Up to Minkowski similarities there are only two Minkowski PH planar cubics. More precisely, any planar Minkowski PH cubic can be mapped by some element of $C O\left(\mathbb{R}^{1,1}\right)$ on one of the following cubics - see Fig. 3.

$$
\begin{aligned}
& \left(\frac{2}{3} t^{3}+2 t, \frac{2}{3} t^{3}-2 t\right)^{\top}, \\
& \left(t+\frac{t^{3}}{3}, t^{2}\right)^{\top} .
\end{aligned}
$$

Proof. Analogously to the proof of Theorem 5, we make use of Lemmas 2 and 4. The preimage of any MPH cubic $\mathbf{p}$ is a straight line $l$, which has one or two intersections with the light cone. If $l$ contains only one intersection, than it is of the form $(a, a)$ or $(a,-a)$ and we apply the mapping $\left(\begin{array}{cc}\frac{1}{a} & 0 \\ 0 & -\frac{1}{a}\end{array}\right)$ or $\left(\begin{array}{cc}\frac{1}{a} & 0 \\ 0 & \frac{1}{a}\end{array}\right)$, respectively, to map this point to the point $(1,-1)$ and the line $l$ to the line given by $(1+t,-1+t)^{\top}$, which gives the cubic (20). If the straight line $l$ has two intersections with the light cone, they are of the form $(a, a)$ and $(b,-b)$. The mapping

$$
\left(\begin{array}{l}
\left(\frac{1}{2 a}+\frac{1}{2 b}\right)\left(\frac{1}{2 a}-\frac{1}{2 b}\right) \\
\left(\frac{1}{2 a}-\frac{1}{2 b}\right)\left(\frac{1}{2 a}+\frac{1}{2 b}\right)
\end{array}\right)
$$

maps the line $l$ on the line given by $(1, t)^{\top}$, which gives the cubic (21).

\section{Planar PH and MPH quintics}

\subsection{PH quintics}

Let $\mathbf{p}(t)$ be a planar quintic in $\mathbb{R}^{2}$ expressed in the Bernstein-Bézier representation [19]

$$
\mathbf{p}(t)=\sum_{i=0}^{5} \mathbf{p}_{i} B_{i}^{5}(t), \quad t \in[0,1]
$$



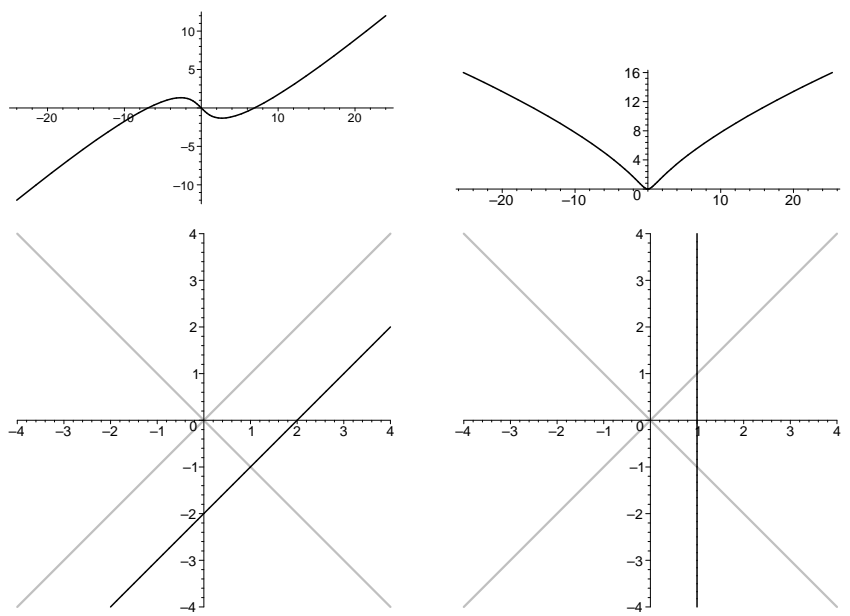

Fig. 3. Two Minkowski PH cubics (top) and their preimages (bottom).

where $\mathbf{p}_{i}$, are the control points. Moreover, let $L_{i}, i=1, \ldots, 5$ be the lengths of the control polygon legs and $\theta_{i}, i=1, \ldots, 4$ be the control polygon angles included at the interior vertices $\mathbf{p}_{i}$. Then, as observed in [10], Section 5, the following constraint on the lengths of the control polygon legs holds for any planar PH quintic

$$
L_{1} L_{4}^{2}=L_{5} L_{2}^{2} .
$$

Using the PH representation map (see [6]) and the complex numbers setting simplifies the matter. We express the hodograph $\mathbf{h}(t)$ of $\mathbf{p}(t)$ and its preimage $\mathcal{A}(t)$ in the Bernstein-Bézier representation

$$
\mathbf{h}(t)=\sum_{i=0}^{4} \mathbf{h}_{i} B_{i}^{4}(t), \quad \mathcal{A}(t)=\sum_{i=0}^{2} \mathcal{A}_{i} B_{i}^{2}(t), \quad t \in[0,1],
$$

where $\mathbf{h}_{i}$ and $\mathcal{A}_{i}$ are the complex control points.

The relation between the hodograph and the preimage

$$
\mathbf{h}(t)=\mathcal{A}(t)^{2}
$$

can be expressed using the control points as

$$
\begin{aligned}
& \mathbf{h}_{0}=\mathcal{A}_{0}^{2}, \\
& \mathbf{h}_{1}=\mathcal{A}_{0} \mathcal{A}_{1}, \\
& \mathbf{h}_{2}=\frac{2}{3} \mathcal{A}_{1}^{2}+\frac{1}{3} \mathcal{A}_{0} \mathcal{A}_{2}, \\
& \mathbf{h}_{3}=\mathcal{A}_{1} \mathcal{A}_{2}, \\
& \mathbf{h}_{4}=\mathcal{A}_{2}^{2} .
\end{aligned}
$$

From the basic properties of complex numbers it follows that

$$
\mathbf{h}_{0} \mathbf{h}_{3}^{2}=\mathbf{h}_{1}^{2} \mathbf{h}_{4},
$$




$$
3 \mathbf{h}_{2} \mathbf{h}_{3} \mathbf{h}_{4}=2 \mathbf{h}_{3}^{3}+\mathbf{h}_{1} \mathbf{h}_{4}^{2}
$$

and

$$
3 \mathbf{h}_{2} \mathbf{h}_{1} \mathbf{h}_{0}=2 \mathbf{h}_{1}^{3}+\mathbf{h}_{3} \mathbf{h}_{0}^{2} .
$$

Note the symmetry of the last two equations. Moreover, a simple computation reveals that (25) can be obtained from (23) and (24). More precisely, expressing e.g. $\mathbf{h}_{0}$ from (23) and substituting it into (24) yields (25).

Now, let $\phi_{i}, i=0, \ldots, 4$ be the argument of $\mathbf{h}_{i}$ in its polar form and $\left|\mathbf{h}_{i}\right|$ its usual complex norm. Then (23) is equivalent to the system

$$
\begin{aligned}
\left|\mathbf{h}_{0}\right|\left|\mathbf{h}_{3}\right|^{2} & =\left|\mathbf{h}_{4}\right|\left|\mathbf{h}_{1}\right|^{2}, \\
\phi_{0}+2 \phi_{3} & =\phi_{4}+2 \phi_{1} .
\end{aligned}
$$

In turn, this system is equivalent to

$$
\begin{aligned}
& L_{1} L_{4}^{2}=L_{5} L_{2}^{2}, \\
& \theta_{2}+\theta_{3}=\theta_{1}+\theta_{4},
\end{aligned}
$$

which yields a geometric constraint on the control polygon of a planar PH quintic. However, it seems that (24) (or (25)) does not admit a straightforward geometric interpretation.

Theorem 10. Let $\mathbf{p}(t)$ be a quintic in $\mathbb{R}^{2}$. Then $\mathbf{p}(t)$ is a PH quintic if and only if

$$
\begin{aligned}
& \mathbf{h}_{0} \mathbf{h}_{3}^{2}=\mathbf{h}_{1}^{2} \mathbf{h}_{4}, \\
& 3 \mathbf{h}_{2} \mathbf{h}_{3} \mathbf{h}_{4}=2 \mathbf{h}_{3}^{3}+\mathbf{h}_{1} \mathbf{h}_{4}^{2} .
\end{aligned}
$$

Proof. It only remains to show the sufficiency of the system (26). Let $\mathbf{p}(t)$ be a planar quintic with its hodograph control points $\mathbf{h}_{i}, i=0, \ldots, 4$ such that it satisfies (26). First, compute $\mathcal{A}_{0}$ from the first equation in (10) using any complex square root. Then, compute $\mathcal{A}_{1}$ from $\mathbf{h}_{1}=\mathcal{A}_{0} \mathcal{A}_{1}$ and $\mathcal{A}_{2}$ from $\mathbf{h}_{3}=\mathcal{A}_{1} \mathcal{A}_{2}$. Thus we have

$$
\mathcal{A}_{0}^{2}=\mathbf{h}_{0}, \quad \mathcal{A}_{1}=\frac{\mathbf{h}_{1}}{\mathcal{A}_{0}}, \quad \mathcal{A}_{2}=\frac{\mathbf{h}_{3}}{\mathcal{A}_{1}} .
$$

Then, using the first equation of (26), we verify that

$$
\mathcal{A}_{2}^{2}=\frac{\mathbf{h}_{3}^{2}}{\mathcal{A}_{1}^{2}}=\frac{\mathbf{h}_{3}^{2} \mathbf{h}_{0}}{\mathbf{h}_{1}^{2}}=\mathbf{h}_{4}
$$

and using the second equation of (26) we see that

$$
\frac{2}{3} \mathcal{A}_{1}^{2}+\frac{1}{3} \mathcal{A}_{0} \mathcal{A}_{2}=\frac{1}{3}\left(2 \frac{\mathbf{h}_{1}^{2}}{\mathbf{h}_{0}}+\frac{\mathbf{h}_{0} \mathbf{h}_{3}}{\mathbf{h}_{1}}\right)=\mathbf{h}_{2} .
$$

Therefore, we found the control points of the preimage of $\mathbf{p}(t)$. Consequently, $\mathbf{p}(t)$ is a planar PH quintic.

Now, we will provide a classification of Euclidean PH quintics, which is based on the methods developed in Section 3. 
Theorem 11. Any planar Euclidean PH quintic can be transformed by some element of $C O\left(\mathbb{R}^{2}\right)$ and a reparameterization to one of the following forms

$$
\begin{gathered}
\left(-\frac{1}{5} t^{5}+\left(\frac{1}{3}-\frac{2}{3} b\right) t^{3}+t^{2} a+\left(a^{2}-b^{2}\right) t, \frac{1}{2} t^{4}+t^{2} b+\frac{2}{3} t^{3} a+2 a b t\right)^{\top} \\
\left(-\frac{1}{5} t^{5}-\frac{2}{3} t^{3} c+\left(1-c^{2}\right) t, \frac{2}{3} t^{3}+2 c t\right)^{\top}
\end{gathered}
$$

where $a \geq 0, b, c$ are real parameters.

Proof. Let $\mathbf{p}$ be a quintic $\mathrm{PH}$ curve and $\mathcal{A}(t)=(u(t), v(t))^{\top}$ its quadratic preimage. From the geometrical point of view there are two possibilities: $\mathcal{A}(t)$ is either a parabola, or it is degenerated into a half-line. In the former case we can rotate the parabola so that its axis has the direction given by $(0,1)^{\top}$. If its vertex $x$-coordinate is negative we apply the mapping $\left(\begin{array}{rr}-1 & 0 \\ 0 & 1\end{array}\right)$ to make it positive. Then, we scale the parabola and reparameterize it so that the vertex corresponds to $t=0$ and it takes the form $\left(t+a, t^{2}+\right.$ $b$ ). This preimage provides the PH curve (27). It follows from Theorem 1 and Lemma 4 that all the used transformations of the preimage will induce a similarity (element of $C O\left(\mathbb{R}^{2}\right)$ transforming $\mathbf{p}(t)$ to the desired form.

If $\mathcal{A}$ degenerates into a half-line, it is of the form $\left(a^{\prime}+k q(t), b^{\prime}+l q(t)\right)^{\top}$, where $q(t)$ is a quadratic polynomial and $a^{\prime}, b^{\prime}, l, k$ some real numbers. Again, we rotate $\mathcal{A}$ so that it points in the direction of $(0,1)^{\top}$ and possibly apply $\left(\begin{array}{rr}-1 & 0 \\ 0 & 1\end{array}\right)$ in order to make the $x$-coordinate of its extremal point positive (it can not be 0 , since this would lead to a degeneration of the quintic $\mathbf{p}$ into a line). Then, we scale it so that the $x$-coordinate of this point is equal to 1 . Eventually, via a suitable reparameterization this half-line will take the form $\left(1, t^{2}+c\right)^{\top}$, which provides the PH curve (28).

The explicit forms of PH quintics (27) and (28) seem to be promising for the analysis of possible singularities and other important features of $\mathrm{PH}$ quintics. In this paper we will restrict ourselves to a description of symmetric $\mathrm{PH}$ curves, which can be obtained from (28) and (27) by setting $a=0$.

Theorem 12. Any PH quintic symmetric with respect to an axis is similar to

$$
\left(-\frac{1}{5} t^{5}+\left(\frac{1}{3}-\frac{2}{3} b\right) t^{3}-b^{2} t, \frac{1}{2} t^{4}+t^{2} b\right)^{\top}
$$

for some $b \in \mathbb{R}$ and any centrally symmetric $P H$ quintic is similar to

$$
\left(-\frac{1}{5} t^{5}-\frac{2}{3} t^{3} c+\left(1-c^{2}\right) t, \frac{2}{3} t^{3}+2 c t\right)^{\top}
$$

for some $c \in \mathbb{R}$.

Proof. Obviously, (29) is symmetric with respect to the $y$ axis and (30) with respect to the origin. Due to Theorem 11 we only need to show that for $a \neq 0$ the curve (27) is not symmetric. However, this follows from the non-symmetric position of the point on the parabola which is closest to the origin. Indeed, for its image on the PH curve there can not be any symmetric point. 

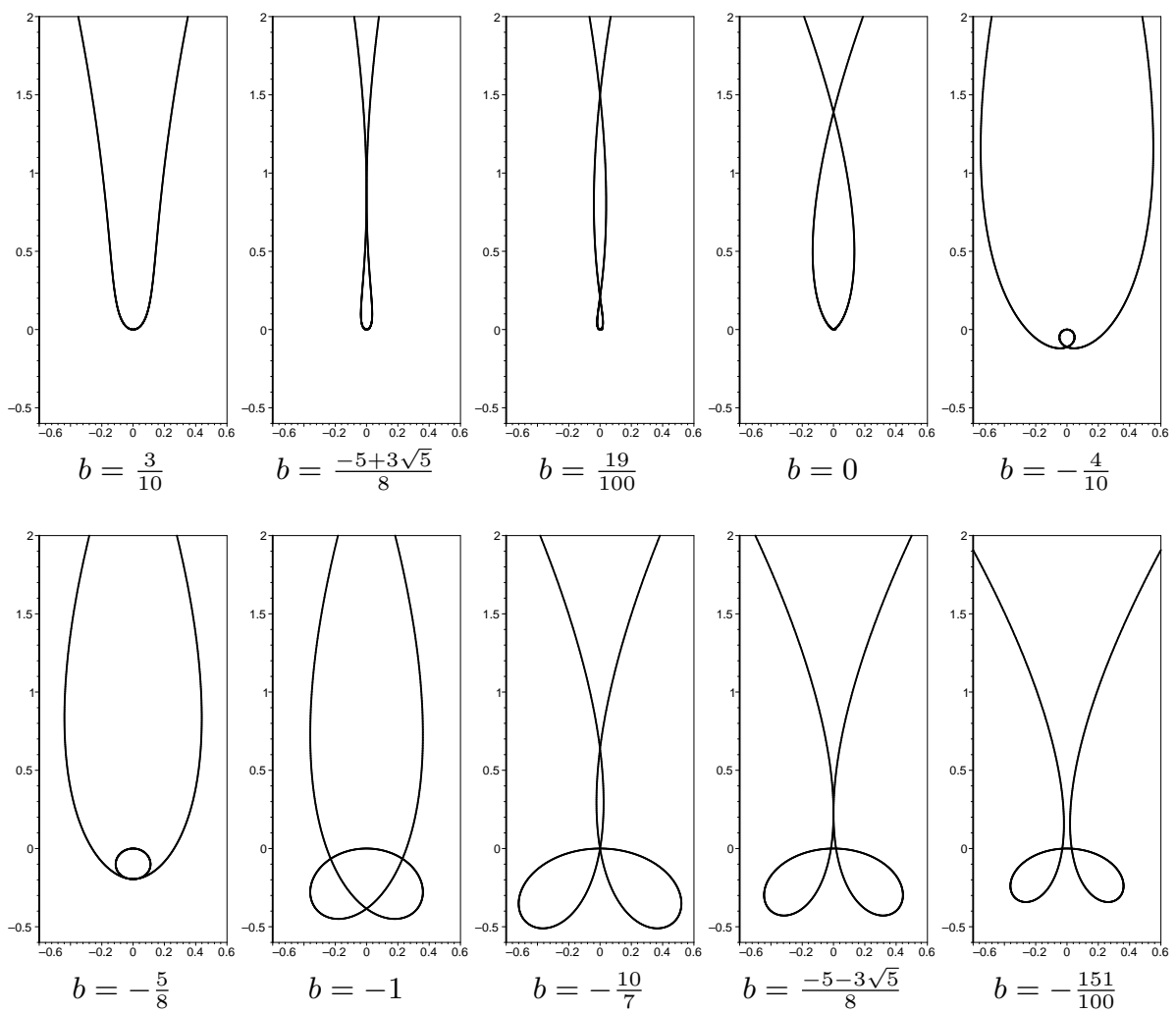

Fig. 4. Examples of $\mathrm{PH}$ quintics symmetric with respect to an axis.

Thus, we see that symmetric PH quintics form two one-parameter systems. Figures 4 and 5 show representatives of these systems for some typical and critical values of the parameters.

\subsection{MPH quintics}

Now, we move on to planar MPH quintics. Using the PH representation map and the Clifford algebra $\mathcal{C}(1,1)$ setting we express planar MPH quintics $\mathbf{p}(t)$, their hodographs $\mathbf{h}(t)$ and the preimages $\mathcal{A}(t)$ as

$$
\mathbf{p}(t)=\sum_{i=0}^{5} \mathbf{p}_{i} B_{i}^{5}(t), \quad \mathbf{h}(t)=\sum_{i=0}^{4} \mathbf{h}_{i} B_{i}^{4}(t), \quad \mathcal{A}(t)=\sum_{i=0}^{2} \mathcal{A}_{i} B_{i}^{2}(t), \quad t \in[0,1],
$$

where $\mathbf{p}_{i}, \mathbf{h}_{i}$ and $\mathcal{A}_{i}$ are the control points. In this setting, $\mathbf{p}(t)$ and its hodograph are described by pure vectors, whereas the preimage lies in the subalgebra $\mathbb{H}^{1,1}$. The relation between the hodograph and the preimage

$$
\mathbf{h}(t)=\mathcal{A}(t) \star \mathcal{A}(t)
$$



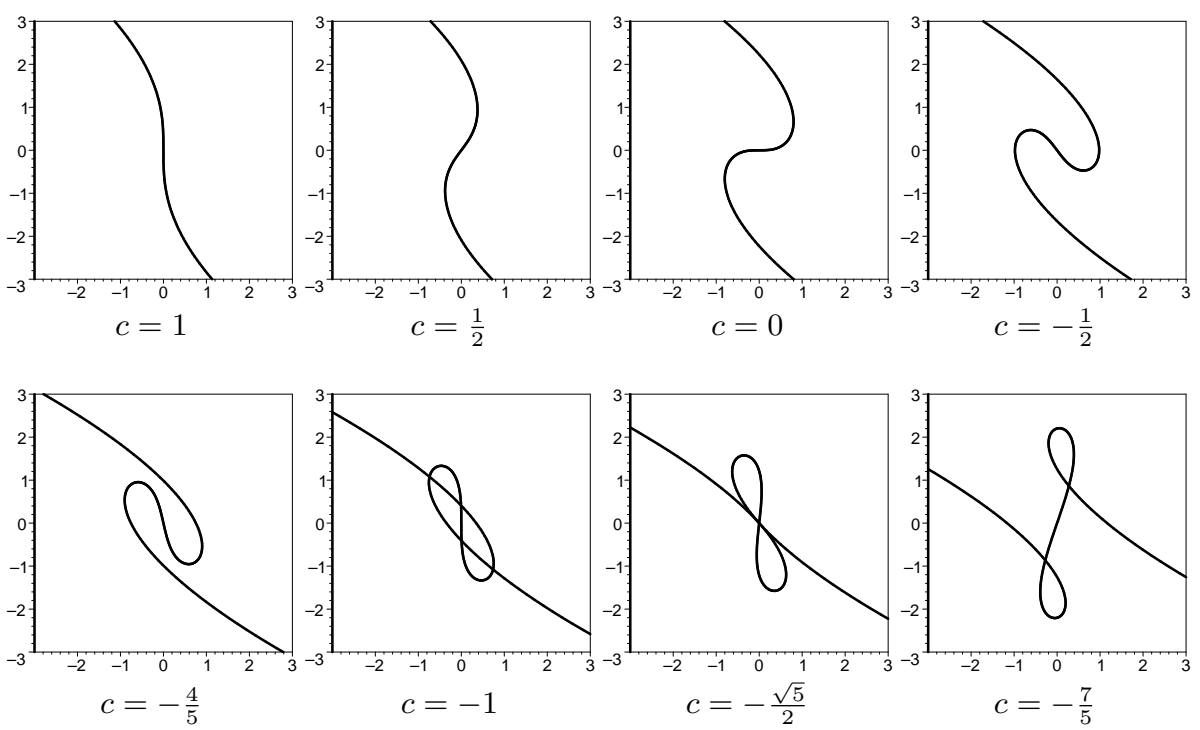

Fig. 5. Examples of PH quintics symmetric with respect to a point.

can be expressed using the control points as

$$
\begin{aligned}
& \mathbf{h}_{0}=\mathcal{A}_{0}^{2 \star} \\
& \mathbf{h}_{1}=\mathcal{A}_{0} \star \mathcal{A}_{1}, \\
& \mathbf{h}_{2}=\frac{2}{3} \mathcal{A}_{1}^{2 \star}+\frac{1}{3} \mathcal{A}_{0} \star \mathcal{A}_{2}, \\
& \mathbf{h}_{3}=\mathcal{A}_{1} \star \mathcal{A}_{2}, \\
& \mathbf{h}_{4}=\mathcal{A}_{2}^{2 \star}
\end{aligned}
$$

Exploiting the properties of the symmetric product $\star$ as in the cubic case, one can see that

$$
\mathbf{h}_{0} \star \mathbf{h}_{3}^{2 \star}=\mathbf{h}_{1}^{2 \star} \star \mathbf{h}_{4},
$$

and

$$
3 \mathbf{h}_{2} \star \mathbf{h}_{3} \star \mathbf{h}_{4}=2 \mathbf{h}_{3}^{3 \star}+\mathbf{h}_{1} \star \mathbf{h}_{4}^{2 \star} .
$$

The symmetric equation holds

$$
3 \mathbf{h}_{2} \star \mathbf{h}_{1} \star \mathbf{h}_{0}=2 \mathbf{h}_{1}^{3 \star}+\mathbf{h}_{3} \star \mathbf{h}_{0}^{2 \star},
$$

but can be derived from (32) and (33). Moreover, using the same techniques as in Section 4.2 , one can show that provided that $\mathbf{h}_{i}, i=0, \ldots, 4$ are positive space-like vectors, (32) is equivalent to the system

$$
\begin{aligned}
& L_{1} L_{4}^{2}=L_{5} L_{2}^{2}, \\
& \theta_{2}+\theta_{3}=\theta_{1}+\theta_{4},
\end{aligned}
$$



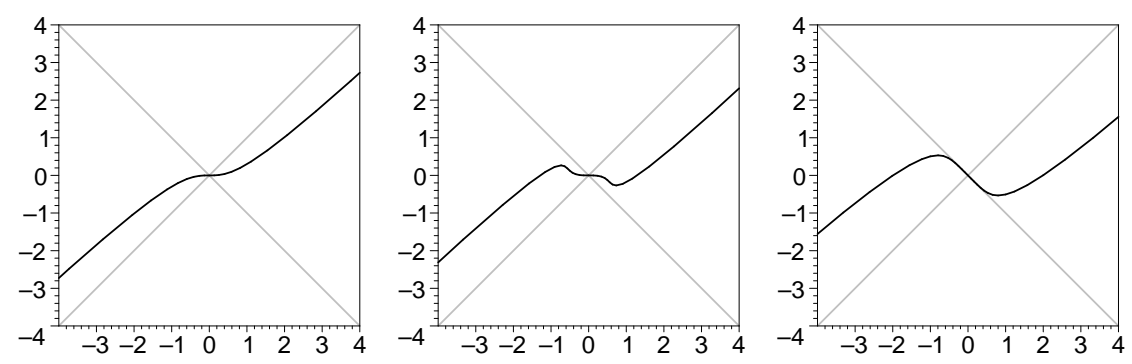

Fig. 6. Shapes of Minkowski PH quintics (42), (43) and (44).

where $L_{i}, i=1, \ldots, 5$ are the Minkowski lengths of the control polygon legs and $\theta_{i}$, $i=1 \ldots, 4$ are the (Minkowski) angles (see Definition 4 ) at the interior vertices of the control polygon of $\mathbf{p}(t)$.

Theorem 13. Let $\mathbf{p}(t)$ be a quintic in $\mathbb{R}^{2}$ such that $\mathbf{h}_{0}$ and $\mathbf{h}_{1}$ are space-like or $\mathbf{h}_{3}$ and $\mathbf{h}_{4}$ are space-like. Then $\mathbf{p}(t)$ is an MPH quintic if and only if

$$
\begin{aligned}
& \mathbf{h}_{0} \star \mathbf{h}_{3}^{2 \star}=\mathbf{h}_{1}^{2 \star} \star \mathbf{h}_{4}, \\
& 3 \mathbf{h}_{2} \star \mathbf{h}_{3} \star \mathbf{h}_{4}=2 \mathbf{h}_{3}^{3 \star}+\mathbf{h}_{1} \star \mathbf{h}_{4}^{2 \star} .
\end{aligned}
$$

Proof. It only remains to show the sufficiency of the system (35). Let $\mathbf{p}(t)$ be a planar quintic with its hodograph control points $\mathbf{h}_{i}, i=0, \ldots, 4$ such that it satisfies (35) and let $\mathbf{h}_{0}$ and $\mathbf{h}_{1}$ be space-like, i.e., invertible in $\mathcal{C}(1,1)$. First, compute $\mathcal{A}_{0}$ from the first equation in (31) using Lemma 2. Then, compute $\mathcal{A}_{1}$ from $\mathbf{h}_{1}=\mathcal{A}_{0} \star \mathcal{A}_{1}$ and $\mathcal{A}_{2}$ from $\mathbf{h}_{3}=\mathcal{A}_{1} \star \mathcal{A}_{2}$. Thus we have (cf. (15))

$$
\mathcal{A}_{0}^{2 \star}=\mathbf{h}_{0}, \quad \mathcal{A}_{1}=\frac{\mathbf{h}_{1} \cdot \mathcal{A}_{0} \cdot \mathbf{e}_{1}}{N\left(\mathcal{A}_{0}\right)}, \quad \mathcal{A}_{2}=\frac{\mathbf{h}_{3} \cdot \mathcal{A}_{1} \cdot \mathbf{e}_{1}}{N\left(\mathcal{A}_{1}\right)} .
$$

Now, using the following formulas

$$
\mathcal{A}_{1}^{2 \star}=\frac{\mathbf{h}_{1} \cdot \mathbf{h}_{0} \cdot \overline{\mathbf{h}}_{1}}{N\left(\mathbf{h}_{0}\right)}, \quad N\left(\mathcal{A}_{1}\right)=-\frac{N\left(\mathbf{h}_{1}\right)}{N\left(\mathcal{A}_{0}\right)}
$$

we compute

$$
\mathcal{A}_{2}^{2 \star}=\frac{\mathbf{h}_{3} \cdot \mathcal{A}_{1} \cdot \mathbf{e}_{1} \cdot \mathbf{e}_{1} \cdot \overline{\mathbf{e}}_{1} \cdot \overline{\mathcal{A}}_{1} \cdot \overline{\mathbf{h}}_{3}}{N\left(\mathcal{A}_{1}\right)^{2}}=-\frac{\mathbf{h}_{3} \cdot \mathcal{A}_{1}^{2 \star} \cdot \overline{\mathbf{h}}_{3}}{N\left(\mathcal{A}_{1}\right)^{2}}=\frac{\mathbf{h}_{3} \cdot \mathbf{h}_{1} \cdot \mathbf{h}_{0} \cdot \overline{\mathbf{h}}_{1} \cdot \overline{\mathbf{h}}_{3}}{N\left(\mathbf{h}_{1}\right)^{2}} .
$$

Verifying that

$$
\mathbf{h}_{1}^{2 \star} \star\left(\mathcal{A}_{2}^{2 \star}\right)=\mathbf{h}_{1}^{2 \star} \star \mathbf{h}_{4},
$$

using the first equation of (35) and the invertibility of $\mathbf{h}_{1}$ we conclude that

$$
\mathcal{A}_{2}^{2 \star}=\mathbf{h}_{4} .
$$

Second, we need to show that

$$
\frac{2}{3} \mathcal{A}_{1}^{2 \star}+\frac{1}{3} \mathcal{A}_{0} \star \mathcal{A}_{2}=\mathbf{h}_{2} .
$$


In order to do that, we compute

$$
\begin{aligned}
& \mathcal{A}_{2}=\frac{\mathbf{h}_{3} \cdot \mathcal{A}_{1} \cdot \mathbf{e}_{1}}{N\left(\mathcal{A}_{1}\right)}=\frac{\mathbf{h}_{3} \cdot \mathbf{h}_{1} \cdot \mathcal{A}_{0}}{N\left(\mathcal{A}_{0}\right) N\left(\mathcal{A}_{1}\right)} \\
& \mathcal{A}_{0} \star \mathcal{A}_{2}=\frac{\mathcal{A}_{0} \cdot \mathbf{e}_{1} \cdot \overline{\mathcal{A}}_{0} \cdot \overline{\mathbf{h}}_{1} \cdot \overline{\mathbf{h}}_{3}+\mathbf{h}_{3} \cdot \mathbf{h}_{1} \cdot \mathcal{A}_{0} \cdot \mathbf{e}_{1} \cdot \overline{\mathcal{A}}_{0}}{2 N\left(\mathcal{A}_{0}\right) N\left(\mathcal{A}_{1}\right)}=\frac{\mathbf{h}_{0} \cdot \overline{\mathbf{h}}_{1} \cdot \overline{\mathbf{h}}_{3}+\mathbf{h}_{3} \cdot \mathbf{h}_{1} \cdot \mathbf{h}_{0}}{2 N\left(\mathcal{A}_{0}\right) N\left(\mathcal{A}_{1}\right)}
\end{aligned}
$$

Thus we have

$$
\frac{2}{3} \mathcal{A}_{1}^{2 \star}+\frac{1}{3} \mathcal{A}_{0} \star \mathcal{A}_{2}=\frac{2 \mathbf{h}_{1} \cdot \mathbf{h}_{0} \cdot \overline{\mathbf{h}}_{1}}{3 N\left(\mathbf{h}_{0}\right)}+\frac{\mathbf{h}_{0} \cdot \overline{\mathbf{h}}_{1} \cdot \overline{\mathbf{h}}_{3}+\mathbf{h}_{3} \cdot \mathbf{h}_{1} \cdot \mathbf{h}_{0}}{6 N\left(\mathcal{A}_{0}\right) N\left(\mathcal{A}_{1}\right)} .
$$

Finally, by a straightforward computation we arrive at

$$
\left(\frac{2}{3} \mathcal{A}_{1}^{2 \star}+\frac{1}{3} \mathcal{A}_{0} \star \mathcal{A}_{2}\right) \star \mathbf{h}_{1} \star \mathbf{h}_{0}=2 \mathbf{h}_{1}^{3 \star}+\mathbf{h}_{3} \star \mathbf{h}_{0}^{2 \star} .
$$

Comparing this equation with (34) gives (36) since $\mathbf{h}_{0}$ and $\mathbf{h}_{1}$ are invertible. Thus we found the control points of the preimage of $\mathbf{p}(t)$. Consequently, $\mathbf{p}(t)$ is a planar MPH quintic. If $\mathbf{h}_{3}$ and $\mathbf{h}_{4}$ are space-like, we simply use the symmetry of the equations (32), (33) and (34).

Now, making use of the methods developed in Section 3, we will give a classification of Minkowski PH quintics.

Theorem 14. Any planar Minkowski PH quintic can be transformed by some element of $C O\left(\mathbb{R}^{1,1}\right)$ and a reparameterization to one of the following forms

$$
\begin{aligned}
& \left(\frac{1}{5} t^{5}+\left(\frac{1}{3}+\frac{2}{3} b\right) t^{3}+t^{2} a+\left(a^{2}+b^{2}\right) t, \frac{1}{2} t^{4}+t^{2} b+\frac{2}{3} t^{3} a+2 a b t\right)^{\top}, \\
& \left(\frac{1}{5} t^{5}+\frac{2}{3} t^{3} c+\left(1+c^{2}\right) t, \frac{2}{3} t^{3}+2 c t\right)^{\top}, \\
& \left(\frac{2}{5} t^{5}+\left(\frac{2}{3}+\frac{4}{3} c\right) t^{3}+2 c^{2} t, \frac{2}{5} t^{5}+\left(-\frac{2}{3}+\frac{4}{3} c\right) t^{3}+2 c^{2} t\right)^{\top}, \\
& \left(\frac{2}{5} t^{5}+\left(\frac{2}{3}+\frac{2}{3} c\right) t^{3}-c t^{2}+c^{2} t, \frac{2}{5} t^{5}+\left(-\frac{2}{3}+\frac{2}{3} c\right) t^{3}+c t^{2}\right)^{\top}, \\
& \left(\frac{2}{5} t^{5}+\frac{2}{3} t^{3}-2 d t^{2}+2 d^{2} t, \frac{2}{5} t^{5}-\frac{2}{3} t^{3}+2 d t^{2}-2 d^{2} t\right)^{\top}, \\
& \left(\frac{2}{5} t^{5}+\frac{2}{3} t^{3}+t, \frac{2}{5} t^{5}+\frac{2}{3} t^{3}-t\right)^{\top}, \\
& \left(\frac{2}{5} t^{5}-\frac{2}{3} t^{3}+t, \frac{2}{5} t^{5}-\frac{2}{3} t^{3}-t\right)^{\top}, \\
& \left(\frac{2}{5} t^{5}+2 t, \frac{2}{5} t^{5}-2 t\right)^{\top},
\end{aligned}
$$

where $a \geq 0, b, c$ and $d>0$ are real parameters. 
Proof. The proof is analogous to the proof of Theorem 11. Let $\mathbf{p}$ be a quintic MPH curve and $\mathcal{A}(t)=(u(t), v(t))^{\top}$ its quadratic preimage. If the preimage is a parabola whose axis has a non-isotropic direction, then via the action of the group $P C O\left(\mathbb{R}^{1,1}\right)$ and a reparameterization it can be transformed into the form $\left(t+a, t^{2}+b\right)$, which gives the quintic (37). If $\mathcal{A}$ degenerates into a half-line with a non-isotropic direction, it can be transformed into the form $\left(1, t^{2}+c\right)^{\top}$, which provides the MPH curve (38).

The remaining cases correspond to the parabola with its axis direction given by $(1,1)^{\top}$ or a half line with the same direction. This orientation can not be changed by any element of $P C O\left(\mathbb{R}^{1,1}\right)$ to a non-isotropic one. These elements can however move the vertex (the half-line end point) of the preimage so that it lies on one of the lines $u=v, v=0$ or $u=-v$. After a scaling and a reparameterization we obtain the following possible preimages for the parabola: $\left(c-t+t^{2}, c+t+t^{2}\right)^{\top}$ giving (39), $\left(c-t+t^{2}, t+t^{2}\right)^{\top}$ yielding (40) and $\left(d-t+t^{2},-d+t+t^{2}\right)^{\top}$ giving (41). In the case of half-line, a suitable scaling removes any remaining free parameters and we arrive at three cases of preimages: $\left(1+t^{2}, t^{2}\right)^{\top}$ giving (42), $\left(-1+t^{2}, t^{2}\right)^{\top}$, which yields (43) and $\left(1+t^{2},-1+t^{2}\right)^{\top}$, which gives (44).

As we have seen in the proof, the main classes of Minkowski PH quintic are (37), (38). The other cases have preimages with particular positions with respect to the isotropic lines. To conclude this section we give figures of the three particular quintics (42)-(44), which are the only centrally symmetrical Minkowski PH quintics having preimages parallel to isotropic lines - see Fig. 6.

\section{Conclusion}

The main contributions of this paper are of two types. First, in the case of Euclidean PH cubics we provide new simple proofs for the known facts [10] about their control polygons and their classification. For Minkowski PH curves the classification result was already published in [23]. We give a simplified proof. Moreover, we give an original sufficient and necessary condition for the control polygon. Results for quintics in both Euclidean and Minkowski planes are new; we provide classifications and sufficient and necessary conditions for control polygons of quintic PH curves.

Second, the concept of the preimage of a transformation was introduced in a general way and it seems to be very promising for studying PH curves of even higher degrees. In particular we plan to give an analysis of possible configurations of singularities for planar PH quintics and to classify low degree curves in the Euclidean and Minkowski three dimensional spaces.

Acknowledgment Zbyněk Šír was supported by the project MSM 0021620839 of the Czech Ministry of Education. We thank all referees for their comments which helped us to improve the paper.

\section{References}

1. Ahn, M.-H., Kim, G.-I. and Lee, C.-N., Geometry of root-related parameters of PH curves. Appl. Math. Lett. 16 (2003), no. 1, 49-57. 
2. Aigner, M., Šír, Z., Jüttler, B. (2007), Evolution-based least-squares fitting using Pythagorean hodograph spline curves, Comput. Aided Geom. Design, 24, 310-322.

3. Cho H. Ch., Choi, H. I., Kwon S.-H., Lee D. S., and Wee N.-S., Clifford algebra, Lorentzian geometry and rational parametrization of canal surfaces, Comp. Aided Geom. Design, 21 (2004), 327-339.

4. Choi, H.I., Han, C.Y., Euler Rodrigues frames on spatial Pythagorean-hodograph curves, Comput. Aided Geom. Design 19 (2002) 603-620.

5. Choi, H. I., Han, Ch. Y., Moon, H. P., Roh, K. H., and Wee, N. S., Medial axis transform and offset curves by Minkowski Pythagorean hodograph curves, Comp.-Aided Design, 31 (1999), 59-72.

6. Choi, H. I., Lee, D. S., and Moon, H. P., Clifford algebra, spin representation and rational parameterization of curves and surfaces, Advances in Computational Mathematics, 17 (2002), $5-48$.

7. Dietz, R., Hoschek, J., Jüttler, B., An algebraic approach to curves and surfaces on the sphere and on other quadrics, Comp. Aided Geom. Design 10 (1993), 211-229.

8. R.T. Farouki (1994), The conformal map $z \rightarrow z^{2}$ of the hodograph plane. Comp. Aided Geom. Design 11, 363-390.

9. Farouki, R.T., and Neff, C.A., (1995), Hermite interpolation by Pythagorean-hodograph quintics. Math. Comput. 64, 1589-1609.

10. Farouki, R. T. and Sakkalis, T. (1990), Pythagorean hodographs, IBM Journal of Research and Development 34, 736-752.

11. Farouki, R.T. and Sakkalis, T., Pythagorean-hodograph space curves, Adv. Comput. Math. 2 (1994) 41-66.

12. Farouki, R.T., Manjunathaiah, J. and Jee, S. (1998), Design of rational cam profiles with Pythagorean-hodograph curves. Mech. Mach. Theory 33, 669-682.

13. Farouki, R.T., Saitou, K. and Tsai,Y-F., (1998), Least-squares tool path approximation with Pythagorean-hodograph curves for high-speed CNC machining. The Mathematics of Surfaces VIII, Information Geometers, Winchester, 245-264.

14. Farouki, R. T., al-Kandari, M., and Sakkalis, T, Hermite interpolation by rotation-invariant spatial Pythagorean-hodograph curves, Advances in Computational Mathematics, 17 (2002), 369-383.

15. Farouki, R. T., Pythagorean-hodograph curves, Handbook of Computer Aided Geometric Design (J. Hoschek, G. Farin and M.-S. Kim, eds.), Elsevier, 2002, 405-427.

16. Farouki, R.T., Manni, C., Sestini, A. (2003), Spatial $C^{2}$ PH quintic splines. Curve and surface design (Saint-Malo, 2002), 147-156, Mod. Methods Math., Nashboro Press.

17. Farouki, R., Pythagorean-Hodograph Curves: Algebra and Geometry Inseparable, Springer, Berlin, 2008, ISBN 978-3-540-73397-3

18. Farouki, R.T., al-Kandari, M. and Sakkalis, T. (2002), Structural invariance of spatial Pythagorean hodographs, Comp. Aided Geom. Design 19, 395-407.

19. Hoschek, J. and Lasser, D. (1996), Fundamentals of Computer Aided Geometric Design, AK Peters, Wellesley MA.

20. Jüttler, B. (2001), Hermite interpolation by Pythagorean hodograph curves of degree seven. Math. Comp. 70, 1089-1111.

21. Jüttler, B., and Mäurer, C., Cubic Pythagorean Hodograph Spline Curves and Applications to Sweep Surface Modeling, Comp.-Aided Design, 31 (1999), 73-83.

22. Kim, G.-I., and Ahn, M.-H. (2003), $C^{1}$ Hermite interpolation using MPH quartic, Comput. Aided Geom. Des. 20, 469-492.

23. Kosinka, J., and Jüttler, B. (2006), Cubic Helices in Minkowski space, Sitzungsber. Österr. Akad. Wiss., Abt. II., 215, 13-35.

24. Kosinka, J., and Jüttler, B., $G^{1}$ Hermite Interpolation by Minkowski Pythagorean Hodograph Cubics, Comp. Aided Geom. Des., 23 (2006), 401-418. 
25. Kosinka, J., and Jüttler, B. (2009), $C^{1}$ Hermite Interpolation by Pythagorean Hodograph Quintics in Minkowski space, Advances in Applied Mathematics 30, 123-140.

26. Kubota, K. K. (1972), Pythagorean triples in unique factorization domains, American Mathematical Monthly 79, 503-505.

27. Lounesto, P., Clifford Algebras and Spinors, London Mathematical Society Lecture Note Series (No. 286), Cambridge University Press, 2001, ISBN-13: 9780521005517.

28. Meek, D.S. and Walton, D.J. (1997), Geometric Hermite interpolation with Tschirnhausen cubics, Journal of Computational and Applied Mathematics 81, 299-309.

29. Moon, H. P. (1999), Minkowski Pythagorean hodographs, Computer Aided Geometric Design 16, 739-753.

30. Moon, H.P., Farouki, R.T and Choi, H.I. (2001), Construction and shape analysis of PH quintic Hermite interpolants, Comp. Aided Geom. Design 18, 93-115.

31. Pottmann, H., and Peternell, M., Applications of Laguerre geometry in CAGD. Comp. Aided Geom. Design, 15 (1997), 165-186.

32. Šír, Z., Jüttler, B. (2005), Euclidean and Minkowski Pythagorean hodograph curves over planar cubics. Comput. Aided Geom. Des. 22, No. 8, 753-770 .

33. Šír, Z., and Jüttler, B., $C^{2}$ Hermite interpolation by spatial Pythagorean hodograph curves, Math. Comp., 76 (2007), 1373-1391.

34. Walton, D.J. and Meek, D.S. (2007), $G^{2}$ curve design with a pair of Pythagorean hodograph quintic spiral segments. (English summary) Comput. Aided Geom. Design 24 , no. 5, $267-$ 285. 